\title{
The prospect of eliminating malaria transmission in some regions of Brazil
}

\author{
Pedro Luiz Tauil/+ \\ Programa de Pós-Graduação em Medicina Tropical, Faculdade de Medicina, Universidade de Brasília, Brasília, DF, Brasil
}

This paper discusses malaria epidemiology and control in Brazil as well as the prospect of interrupting transmission in some areas of the country. The concepts of receptivity and vulnerability of an area to malaria transmission are analysed to predict where elimination might occur in a near future. Outside of the Amazon Region and in the oriental states of the Amazon, such as Tocantins, Maranhão and Mato Grosso, it is likely that malaria transmission can be eliminated with the development and sustained use of a good surveillance system.

Key words: malaria - Brazil - elimination - prospects

Doctor Robert D Newman, director of the World Health Organization's (WHO) Global Malaria Programme, recently stated in the WHO Bulletin (Newman 2011) "that eradicating malaria is the only morally acceptable endgoal, one that will take 40 years or more to achieve. Today, it is possible to reduce malaria in the places where it is worst and to eliminate it from the fringes where it is already low. But it is not possible to take the centre of Africa and reduce malaria to zero with today tools".

The incidence of malaria is influenced by political, economic, social, ecological and biological factors. The absence of effective control measures, such as an efficacious and safe vaccine, also plays a role. For these reasons, one could say that Dr. Newman's statement is also valid in Brazil.

Malaria incidence in Brazil varies throughout the country. For the last 40 years, the disease was concentrated in the Amazonian Basin Region, which includes the states of Acre, Amapá (AP), Amazonas, Maranhão (MA), Mato Grosso (MT), Pará, Rondônia, Roraima and Tocantins (TO). Currently, greater than $99 \%$ of reported cases in the country occur in these states.

The Amazon Region possesses several environmental characteristics that favour the transmission of malaria and make the disease control difficult. The first of these factors is the abundance of the Anopheles (Nyssorhynchus) darlingi mosquito, which is the main malaria vector in the country. Clean, hot and slow-moving water is the main breeding site for the mosquito. In addition, temperatures above $16^{\circ} \mathrm{C}$, low altitude, high humidity and rainfall are all favourable conditions for the survival of the mosquito, which seeks shelter in the surrounding forests. All of these characteristics are found in the Amazon Region and make this region highly receptive to malaria transmission.

+ Corresponding author: pltauil@unb.br

Received 19 April 2011

Accepted 8 June 2011
Related to these environmental factors, there are social and economic determinants of malaria transmission. For example, provisional houses, which are often missing complete exterior walls, are found in areas that have experienced deforestation, recent colonisation and not mechanical mineral exploitation. Without exterior walls, the mechanical barriers to mosquito bites are practically nonexistent. In the absence of complete exterior walls, insecticide spray cannot be applied. In addition, the low population density and long distances between localities make access to these areas difficult and transportation costly.

The cases of malaria have been concentrated in this region since the early 1970 s. These cases have been directly related to the intense, but disorganised, occupation of the region by migrants from other Brazilian regions. These immigrants moved with encouragement from the government and envisioned economic development as the ultimate goal.

Even within the Amazon Region, the incidence levels of malaria vary by location. Malaria is concentrated in areas where groups of people move in search for better living conditions. These areas include the periphery of large cities and sites of recent colonisation, mining, agricultural projects, construction of roads and hydroelectric plants.

In 2007 , more than $80 \%$ of malaria cases were reported in 60 municipalities. This is only $7.4 \%$ of the $807 \mathrm{mu}-$ nicipalities in the Brazilian Amazon Region, according to data from the National Programme of Malaria Control of the Ministry of Health in 2008. This unequal distribution is related to the migration of people to specific regions. In general, areas with high transmission levels are those where people have just recently arrived. In these areas, the houses are precarious and work conditions are poor. The people that move here live next to the forests and water collections that support malaria transmission.

In Brazil, 333,424 malaria cases were reported in 2010 and, of these, 332,310 (99.7\%) were contracted in the Amazon Region. Of the cases reported in the extraAmazon Region, $85 \%$ were imported from the Amazon or from other endemic countries, and only 168 cases were autochthonous. Transmission outside of the Amazon is rare and is restricted to small residual foci or new 
foci with low rates. These remaining disease foci are the result of reintroduction of the parasite from patients who where infected in areas where the vector is still present. In 2010, the states of Espírito Santo, Paraná and São Paulo (SP) reported the majority of the malaria cases $(45,42$ and 20 , respectively).

The present objectives of the National Programme of Malaria Control include interruption of malaria transmission in urban areas of the Amazon Region, which is a prospect that seems to be reachable. At the same time, some states in the eastern Amazon Region (TO, MA and MT) are reporting a decreasing number of new malaria cases every year, which allows us to visualise a possible interruption in transmission in the near future.

In areas where transmission of malaria has already been interrupted, the National Programme aims to keep transmission rates at zero. In the case that outbreaks do occur in these areas, the National Programme aims to use a surveillance system for early detection.

It is worthwhile to remind ourselves of two principles of malaria epidemiology: receptivity and vulnerability. Receptivity refers to the presence of conditions that are ideal for disease transmission, specifically the existence of a minimum density of competent vectors. Vulnerability refers to the probability that infected people from endemic areas enter the region and introduce the parasite.

In Brazil, the receptivity of regions outside of the Amazon is decreasing substantially every year with increasing deforestation and fewer breeding sites for the An. (Nys) darlingi mosquito. In the forests along the Atlantic, particularly in the Southeast and South Regions, from the state of Rio de Janeiro to the north of the state of Rio Grande do Sul, the vector Anopheles (Kerteszia) cruzii still exists. This mosquito proliferates in water collections in bromeliads. Along the coastline, from AP, in the north Region, to SP, it is possible to find the Anopheles (Nyssorhynchus) aquasalis, which breeds in brackish water. Other malaria vectors of the albitarsis species are found in other regions of the country.

Therefore, regions outside of the Amazon do exhibit receptivity and require a sensitive surveillance system to detect early cases of malaria to provide immediate treatment and to prevent local transmission. Areas that are visited by a large number of people from endemic areas become more vulnerable to malaria transmission and must undergo active and effective surveillance. Welltrained individuals who have fieldwork experience are an important resource needed to achieve the objective of interrupting malaria transmission.

In the Amazon Region, urban areas and other important municipalities are highly receptive, as these areas still have forests and breeding sites for mosquitoes primarily in the periphery of the cities. Moreover because of the high rates of travel from rural to urban areas, these areas are still vulnerable to introduction of the parasite by symptomatic and asymptomatic carriers of Plasmodium sp.

Any program of disease control aims to reduce the mortality and severity of disease (Tauil 1998). In the case of malaria, these objectives are being met in Brazil. The number of malaria deaths is decreasing, with less than 100 cases per year in the last five years. Additionally, the rate of hospitalisation due to malaria, a proxy for disease severity, is decreasing; in 2010 there were approximately 5,000 cases.

The National Programme aims to reach these objectives by employing the strategies of early diagnosis and immediate treatment of confirmed cases. Recent data show that more than $60 \%$ of cases start treatment within $48 \mathrm{~h}$ of the onset of symptoms.

More ambitious objectives, such as the reduction of disease incidence, elimination of transmission and eradication are being pursued adding integrated measures of vector control to early detection and treatment of cases looking for reducing the time parasites remains in the blood of people as sources for mosquitoes infections. These methods include the use of impregnated bed nets and improvements in epidemiological surveillance for early detection and treatment. New drugs and shorter treatment schedules are necessary to overcome drug resistance and improve patient compliance.

In the last few years, the incidence of malaria in Brazil has been declining. Disease elimination, which is defined as the absence of autochthonous cases in a predetermined area with the use of control activities (Evans 1985), is being achieved in Brazil outside of the Amazon. Here, only a small number of residual cases are being reported. However, eradication, which is defined as the absence of autochthonous cases in a predetermined area without the use of any control activity (Henderson 1980, Fenner et al. 1988, Forattini 1988, Oliveira-Ferreira et al. 2010), is still a remote objective due to the continued receptivity and vulnerability of a very large portion of Brazil.

\section{REFERENCES}

Evans AS 1985. The eradication of communicable diseases: myth or reality? Am J Epidemiol 122: 199-207.

Fenner F, Henderson DA, Arita I, Jezek Z, Ladnyi ID 1988. Smallpox and its eradication, World Health Organization, Geneva, 1460 pp.

Forattini OP 1988. Varíola, erradicação e doenças infecciosas. Rev Saude Publ 22: 371-374.

Henderson DA 1980. Smallpox eradication. Public Health Rep 95: 422-426.

Newman RD 2011. Learning to outwit malaria. Bull World Health Organ 89: 10-11.

Oliveira-Ferreira J, Lacerda MV, Brasil P, Ladislau JL, Tauil PL, DanielRibeiro CT 2010. Malaria in Brazil: an overview. Malar J 9: 115.

Tauil PL 1998. Controle de agravos à saúde: consistência entre objetivos e medidas preventivas. Inf Epidemiol SUS 7: 55-58. 